\title{
Degenerate, partially degenerate and totally degenerate Daehee numbers and polynomials
}

\section{Dongkyu Lim*}

\section{"Correspondence:}

dgrim84@knu.ac.kr Department of Mathematics, Kyungpook National University, Daegu, 702-701, South Korea

\begin{abstract}
Recently, Kim and Seo considered the partially degenerate Bernoulli numbers and polynomials of the first kind and of the second kind and investigated some properties of these numbers and polynomials. In this paper, we consider degenerate Daehee numbers and polynomials and extend them to partially and totally degenerate Daehee numbers and polynomials. We give some properties and identities and relate those numbers and polynomials with Daehee polynomials and Bernoulli polynomials of the second kind. Some $q$-analogues of the degenerate Bernoulli polynomials are studied by Kim et al. This research influenced us to study $q$-analogues of the degenerate Daehee numbers and polynomials. We also study $q$-analogues of partially and totally degenerate Daehee numbers and polynomials. And we give some formulas related to those numbers and polynomials.
\end{abstract}

MSC: 11B68; 11B75; 11B83; 33E30; 33F99

Keywords: degenerate Daehee polynomial; partially degenerate Daehee polynomial; totally degenerate Daehee polynomial; $q$-analogues of degenerate Daehee polynomial

\section{Introduction}

Let $p$ be a fixed prime number. Throughout this paper, $\mathbb{Z}_{p}, \mathbb{Q}_{p}$ and $\mathbb{C}_{p}$ will denote the ring of $p$-adic integers, the field of $p$-adic rational numbers and the completion of the algebraic closure of $\mathbb{Q}_{p}$, respectively. The $p$-adic norm is normalized as $|p|_{p}=\frac{1}{p}$. Let $\operatorname{UD}\left(\mathbb{Z}_{p}\right)$ be the space of all $\mathbb{C}_{p}$-valued uniformly differentiable functions of $\mathbb{Z}_{p}$.

The ordinary $p$-adic invariant integral on $\mathbb{Z}_{p}$ is given by

$$
I_{0}(f)=\int_{\mathbb{Z}_{p}} f(x) d \mu_{0}(x)=\lim _{N \rightarrow \infty} \frac{1}{p^{N}} \sum_{x=0}^{P^{N}-1} f(x) .
$$

The Daehee polynomials are given by the generating function to be

$$
\sum_{n=0}^{\infty} D_{n}(x) \frac{t^{n}}{n !}=\frac{\log (1+t)}{t}(1+t)^{x} \quad(\text { see }[1,2])
$$

There are many works related with Daehee numbers and polynomials (see [1-8]).

(c) $2015 \mathrm{Lim}$. This article is distributed under the terms of the Creative Commons Attribution 4.0 International License (http://creativecommons.org/licenses/by/4.0/), which permits unrestricted use, distribution, and reproduction in any medium, provided you give appropriate credit to the original author(s) and the source, provide a link to the Creative Commons license, and indicate if changes were made. 
For $\lambda, t \in \mathbb{C}_{p}$ with $|\lambda t|<p^{-\frac{1}{p-1}}$, the Daehee polynomials with parameter $\lambda$ are defined by the generating function to be

$$
\sum_{n=0}^{\infty} D_{n}(x \mid \lambda) \frac{t^{n}}{n !}=\frac{\log (1+\lambda t)^{\frac{1}{\lambda}}}{(1+\lambda t)^{\frac{1}{\lambda}}-1}(1+\lambda t)^{\frac{x}{\lambda}} \quad(\text { see }[9-12]) .
$$

When $\lambda=1, D_{n}(x)=D_{n}(x \mid 1)$ are the Daehee polynomials in $(2)(n \geq 0)$.

As it is well known, Bernoulli polynomials of the first kind $B_{n}(x)$ and of the second kind $b_{n}(x)$ are defined by the generating function to be

$$
\sum_{n=0}^{\infty} B_{n}(x) \frac{t^{n}}{n !}=\frac{t}{e^{t}-1} e^{t x}, \quad \sum_{n=0}^{\infty} b_{n}(x) \frac{t^{n}}{n !}=\frac{t}{\log (1+t)}(1+t)^{x}
$$

respectively (see [13-16]). When $x=0, B_{n}=B_{n}(0)$ and $b_{n}=b_{n}(0)$ are the Bernoulli numbers of the first kind and of the second kind, respectively.

In [17], the degenerate Bernoulli polynomials are defined by Carlitz to be

$$
\sum_{n=0}^{\infty} \beta_{n}(x \mid \lambda) \frac{t^{n}}{n !}=\frac{t}{(1+\lambda t)^{\frac{1}{\lambda}}-1}(1+\lambda t)^{\frac{x}{\lambda}}
$$

Let $q$ be an indeterminate such that $|1-q|_{p}<p^{-\frac{1}{p-1}}$. The $q$-extension of the number $x$ is defined as $[x]_{q}=\frac{1-q^{x}}{1-q}$. Note that $\lim _{q \rightarrow 1}[x]_{q}=x$. For $f \in \mathrm{UD}\left(\mathbb{Z}_{p}\right)$, the $p$-adic $q$-integral on $\mathbb{Z}_{p}$ is defined [18] by Kim to be

$$
I_{q}(f)=\int_{\mathbb{Z}_{p}} f(x) d \mu_{q}(x)=\lim _{N \rightarrow \infty} \frac{1}{\left[p^{N}\right]_{q}} \sum_{x=0}^{P^{N}-1} f(x) q^{x} .
$$

From (6), we can derive the following integral equation:

$$
q I_{q}\left(f_{1}\right)-I_{q}(f)=(q-1) f(0)+\frac{q-1}{\log q} f^{\prime}(0) \quad(\text { see }[18])
$$

where $f_{1}(x)=f(x+1)$.

When we study $q$-analogues of Bernoulli polynomials or Daehee polynomials, the above (6) and (7) are very useful (see [10, 13, 19-22]).

Now we recall from [19] that the $q$-analogues of Bernoulli polynomials of the second kind $b_{n, q}(x)$, degenerate Bernoulli polynomials of the first kind $\beta_{n, q}(x \mid \lambda)$, of the second kind $b_{n, q}(x \mid \lambda)$ and $\lambda$-Daehee polynomials $D_{n, q}(x \mid \lambda)$ are generated by

$$
\begin{aligned}
& \sum_{n=0}^{\infty} b_{n, q}(x) \frac{t^{n}}{n !}=\frac{q(1+t)-1}{\log q+\log (1+t)}(1+t)^{x}, \\
& \sum_{n=0}^{\infty} \beta_{n, q}(x \mid \lambda) \frac{t^{n}}{n !}=\frac{t}{q(1+\lambda t)^{\frac{1}{\lambda}}-1}(1+\lambda t)^{\frac{x}{\lambda}} \\
& \sum_{n=0}^{\infty} b_{n, q}(x \mid \lambda) \frac{t^{n}}{n !}=\frac{t}{(q-1)+\frac{q-1}{\log q} \log (1+\lambda t)^{\frac{1}{\lambda}}}(1+\lambda t)^{\frac{x}{\lambda}} \\
& \sum_{n=0}^{\infty} D_{n, q}(x \mid \lambda) \frac{t^{n}}{n !}=\frac{(q-1)+\frac{q-1}{\log q} \log (1+\lambda t)^{\frac{1}{\lambda}}}{q(1+\lambda t)^{\frac{1}{\lambda}}-1}(1+\lambda t)^{\frac{x}{\lambda}}
\end{aligned}
$$


respectively. When $x=0, b_{n, q}=b_{n, q}(0), \beta_{n, q}(\lambda)=\beta_{n, q}(0 \mid \lambda), b_{n, q}(\lambda)=b_{n, q}(0 \mid \lambda)$ and $D_{n, q}(\lambda)=$ $D_{n, q}(0 \mid \lambda)$ are called the $q$-analogues of Bernoulli numbers of the second kind, degenerate Bernoulli numbers of the first kind, of the second kind and $\lambda$-Daehee numbers, respectively. Note that $\lim _{q \rightarrow 1} b_{n, q}(x)=b_{n}(x)$.

The purpose of this paper is to study the degenerate, partially degenerate and totally degenerate Daehee numbers and polynomials. In addition, we study $q$-analogues of those numbers and polynomials.

\section{On degenerate Daehee polynomials}

For $\lambda, t \in \mathbb{C}_{p}$ with $|\lambda t|<p^{-\frac{1}{p-1}}$, we consider the degenerate Daehee polynomials which are given by the generating function to be

$$
\frac{\log (1+t)}{\log (1+\lambda t)^{\frac{1}{\lambda}}}(1+t)^{x}=\sum_{n=0}^{\infty} d_{n}(x \mid \lambda) \frac{t^{n}}{n !}
$$

When $x=0, d_{n}(\lambda)=d_{n}(0 \mid \lambda)$ are called the degenerate Daehee numbers,

$$
\lim _{\lambda \rightarrow 0} \frac{\log (1+t)}{\log (1+\lambda t)^{\frac{1}{\lambda}}}(1+t)^{x}=\frac{\log (1+t)}{t}(1+t)^{x}=\sum_{n=0}^{\infty} D_{n}(x) \frac{t^{n}}{n !}
$$

Thus, $\lim _{\lambda \rightarrow 0} d_{n}(x \mid \lambda)=D_{n}(x)$.

The degenerate Daehee numbers and polynomials are constructed by degenerating only denominator of Daehee numbers and polynomials. This idea comes from the observation of degenerate Bernoulli numbers and polynomials which are defined and studied by Carlitz.

From the degenerate Daehee polynomials in (9),

$$
\begin{aligned}
\sum_{n=0}^{\infty} d_{n}(x \mid \lambda) \frac{t^{n}}{n !} & =\left(\sum_{m=0}^{\infty} d_{m}(\lambda) \frac{t^{m}}{m !}\right)\left(\sum_{l=0}^{\infty}(x)_{l} \frac{t^{l}}{l !}\right) \\
& =\sum_{n=0}^{\infty}\left(\sum_{m=0}^{n}\left(\begin{array}{c}
n \\
m
\end{array}\right) d_{m}(\lambda)(x)_{n-m}\right) \frac{t^{n}}{n !}
\end{aligned}
$$

and

$$
\begin{aligned}
\sum_{n=0}^{\infty} d_{n}(x+y \mid \lambda) \frac{t^{n}}{n !} & =\frac{\log (1+t)}{\log (1+\lambda t)^{\frac{1}{\lambda}}}(1+t)^{x+y} \\
& =\left(\sum_{l=0}^{\infty} d_{l}(x \mid \lambda) \frac{t^{l}}{l !}\right)\left(\sum_{m=0}^{\infty}(y)_{m} \frac{t^{m}}{m !}\right) \\
& =\sum_{n=0}^{\infty}\left(\sum_{l=0}^{n}\left(\begin{array}{l}
n \\
l
\end{array}\right) d_{l}(x \mid \lambda)(y)_{n-l}\right) \frac{t^{n}}{n !} .
\end{aligned}
$$

We note that (11) implies that the degenerate Daehee polynomials are a Sheffer sequence. Thus, from (10) and (11), we have the following theorem. 
Theorem 1 For $n \geq 0$, we have

$$
d_{n}(x \mid \lambda)=\sum_{m=0}^{n}\left(\begin{array}{l}
n \\
m
\end{array}\right) d_{m}(\lambda)(x)_{n-m}
$$

and

$$
d_{n}(x+y \mid \lambda)=\sum_{m=0}^{n}\left(\begin{array}{c}
n \\
m
\end{array}\right) d_{m}(x \mid \lambda)(y)_{n-m}
$$

From (9), we consider the following:

$$
\begin{aligned}
\frac{\log (1+t)}{\log (1+\lambda t)^{\frac{1}{\lambda}}}(1+t)^{x} & =\frac{\log (1+t)}{t} \frac{\lambda t}{\log (1+\lambda t)}(1+t)^{x} \\
& =\left(\sum_{l=0}^{\infty} D_{l}(x) \frac{t^{l}}{l !}\right)\left(\sum_{m=0}^{\infty} B_{m}^{(m)}(1) \frac{\lambda^{m} t^{m}}{m !}\right) \\
& =\sum_{n=0}^{\infty}\left(\sum_{l=0}^{n}\left(\begin{array}{l}
n \\
l
\end{array}\right) D_{l}(x) B_{n-l}^{(n-l)}(1) \lambda^{n-l}\right) \frac{t^{n}}{n !},
\end{aligned}
$$

where $B_{n}^{(r)}(x)$ are the Bernoulli polynomials of order $r$ given by the generating function to be

$$
\left(\frac{t}{e^{t}-1}\right)^{r} e^{x t}=\sum_{n=0}^{\infty} B_{n}^{(r)}(x) \frac{t^{n}}{n !}
$$

By comparing the coefficients of both sides of (12), we have the following result.

Theorem 2 For $n \geq 0$, we have

$$
d_{n}(x \mid \lambda)=\sum_{l=0}^{n}\left(\begin{array}{l}
n \\
l
\end{array}\right) D_{l}(x) B_{n-l}^{(n-l)}(1) \lambda^{n-l} .
$$

From (9), for $\lambda, t \in \mathbb{C}_{p}$ with $|\lambda t|<p^{-\frac{1}{p-1}}$, we observe the following identity:

$$
\begin{aligned}
& \frac{\log (1+t)}{\log (1+\lambda t)^{\frac{1}{\lambda}}}(1+t)^{x} \int_{\mathbb{Z}_{p}}(1+\lambda t)^{\frac{x}{\lambda}} d \mu_{0}(x) \\
& =\left(\sum_{m=0}^{\infty} d_{m}(x \mid \lambda) \frac{t^{m}}{m !}\right)\left(\sum_{l=0}^{\infty} D_{l}(\lambda) \frac{t^{l}}{l !}\right) \\
& =\sum_{n=0}^{\infty}\left(\sum_{m=0}^{n}\left(\begin{array}{c}
n \\
m
\end{array}\right) d_{m}(x \mid \lambda) D_{n-m}(\lambda)\right) \frac{t^{n}}{n !} .
\end{aligned}
$$

On the other hand, by (2) and (5), we have the following:

$$
\begin{gathered}
\frac{\log (1+t)}{\log (1+\lambda t)^{\frac{1}{\lambda}}}(1+t)^{x} \int_{\mathbb{Z}_{p}}(1+\lambda t)^{\frac{x}{\lambda}} d \mu_{0}(x) \\
=\frac{t}{(1+\lambda t)^{\frac{1}{\lambda}}-1} \frac{\log (1+t)}{t}(1+t)^{x}
\end{gathered}
$$




$$
\begin{aligned}
& =\left(\sum_{m=0}^{\infty} \beta_{m}(\lambda) \frac{t^{m}}{m !}\right)\left(\sum_{l=0}^{\infty} D_{l}(x) \frac{t^{l}}{l !}\right) \\
& =\sum_{n=0}^{\infty}\left(\sum_{m=0}^{n}\left(\begin{array}{c}
n \\
m
\end{array}\right) \beta_{m}(\lambda) D_{n-m}(x)\right) \frac{t^{n}}{n !} .
\end{aligned}
$$

Therefore, by (13) and (14), we obtain the following theorem.

Theorem 3 For $n \geq 0$, we have

$$
\sum_{m=0}^{n}\left(\begin{array}{l}
n \\
m
\end{array}\right) d_{m}(x \mid \lambda) D_{n-m}(\lambda)=\sum_{m=0}^{n}\left(\begin{array}{c}
n \\
m
\end{array}\right) \beta_{m}(\lambda) D_{n-m}(x) .
$$

Let us take $\lambda=1$ and replace $t$ by $e^{\left(e^{t}-1\right)}-1$ in (9). Then we have

$$
\begin{aligned}
e^{\left(e^{t}-1\right) x} & =\sum_{n=0}^{\infty} d_{n}(x \mid 1) \frac{1}{n !}\left(e^{\left(e^{t}-1\right)}-1\right)^{n} \\
& =\sum_{n=0}^{\infty} d_{n}(x \mid 1) \frac{1}{n !} n ! \sum_{m=n}^{\infty} S_{2}(m, n) \frac{1}{m !}\left(e^{t}-1\right)^{m} \\
& =\sum_{m=0}^{\infty}\left(\sum_{n=0}^{m} d_{n}(x \mid 1) S_{2}(m, n)\right) \frac{1}{m !}\left(e^{t}-1\right)^{m} \\
& =\sum_{m=0}^{\infty}\left(\sum_{n=0}^{m} d_{n}(x \mid 1) S_{2}(m, n)\right) \frac{1}{m !} m ! \sum_{k=m}^{\infty} S_{2}(k, m) \frac{t^{k}}{k !} \\
& =\sum_{k=0}^{\infty}\left(\sum_{m=0}^{k} \sum_{n=0}^{m} d_{n}(x \mid 1) S_{2}(m, n) S_{2}(k, m)\right) \frac{t^{k}}{k !} .
\end{aligned}
$$

On the other hand,

$$
e^{\left(e^{t}-1\right) x}=\sum_{k=0}^{\infty} \operatorname{Bel}_{k}(x) \frac{t^{k}}{k !},
$$

where $\operatorname{Bel}_{k}(x)$ are called the $k$ th Bell polynomial (see [23]).

Therefore, by (15) and (16), we obtain the following theorem.

Theorem 4 For $n \geq 0$, we have

$$
\operatorname{Bel}_{k}(x)=\sum_{m=0}^{k} \sum_{n=0}^{m} d_{n}(x \mid 1) S_{2}(m, n) S_{2}(k, m) .
$$

For $r \in \mathbb{N}$, we define the higher-order degenerate Daehee polynomials which are given by the generating function to be

$$
\left(\frac{\log (1+t)}{\log (1+\lambda t)^{1 / \lambda}}\right)^{r}(1+t)^{x}=\sum_{n=0}^{\infty} d_{n}^{(r)}(x \mid \lambda) \frac{t^{n}}{n !} .
$$

When $x=0, d_{n}^{(r)}(\lambda)=d_{n}^{(r)}(0 \mid \lambda)$ are called the degenerate Daehee numbers of order $r$. 
From (17), we note that

$$
\begin{aligned}
\sum_{n=0}^{\infty} d_{n}^{(r)}(x \mid \lambda) \frac{t^{n}}{n !} & =\left(\frac{\lambda t}{\log (1+\lambda t)}\right)^{r}\left(\frac{\log (1+t)}{t}\right)^{r}(1+t)^{x} \\
& =\left(\sum_{m=0}^{\infty} B_{m}^{(m-r+1)}(1) \frac{\lambda^{m} t^{m}}{m !}\right)\left(\sum_{l=0}^{\infty} D_{l}^{(r)}(x) \frac{t^{l}}{l !}\right) \\
& =\sum_{n=0}^{\infty}\left(\sum_{m=0}^{n}\left(\begin{array}{c}
n \\
m
\end{array}\right) B_{m}^{(m-r+1)}(1) \lambda^{m} D_{n-m}^{(r)}(x)\right) \frac{t^{n}}{n !}
\end{aligned}
$$

Thus, we have the following theorem.

Theorem 5 For $n \geq 0$, we have

$$
d_{n}^{(r)}(x \mid \lambda)=\sum_{m=0}^{n}\left(\begin{array}{l}
n \\
m
\end{array}\right) B_{m}^{(m-r+1)}(1) \lambda^{m} D_{n-m}^{(r)}(x) .
$$

Specially, for the higher-order degenerate Daehee numbers case, we have the following observation:

$$
\begin{aligned}
\sum_{n=0}^{\infty} d_{n}^{(r)}(\lambda) \frac{t^{n}}{n !} & =\left(\frac{\lambda t}{\log (1+t \lambda)}\right)^{r}\left(\frac{\log (1+t)}{t}\right)^{r} \\
& =\left(\sum_{m=0}^{\infty} B_{m}^{(m-r+1)}(1) \frac{\lambda^{m} t^{m}}{m !}\right)\left(r ! \sum_{l=0}^{\infty} S_{1}(l+r, l) \frac{t^{l}}{(l+r) !}\right) \\
& =\left(\sum_{m=0}^{\infty} B_{m}^{(m-r+1)}(1) \frac{\lambda^{m} t^{m}}{m !}\right)\left(\sum_{l=0}^{\infty} \frac{S_{1}(l+r, r)}{\left(\begin{array}{c}
l+r \\
r
\end{array}\right)} \frac{t^{l}}{l !}\right) \\
& =\sum_{n=0}^{\infty}\left(\sum_{l=0}^{n}\left(\begin{array}{c}
n \\
l
\end{array}\right) \frac{S_{1}(l+r, r)}{\left(\begin{array}{c}
l+r \\
r
\end{array}\right)} \lambda^{n-l} B_{n-l}^{(n-l-r+1)}(1)\right) \frac{t^{n}}{n !} .
\end{aligned}
$$

Thus, by (19), we have the following theorem.

Theorem 6 For $n \geq 0$, we have

$$
d_{n}^{(r)}(\lambda)=\sum_{l=0}^{n}\left(\begin{array}{l}
n \\
l
\end{array}\right) \frac{S_{1}(l+r, r)}{\left(\begin{array}{c}
l+r \\
r
\end{array}\right)} \lambda^{n-l} B_{n-l}^{(n-l-r+1)}(1)
$$

and

$$
d_{n}^{(r)}(x \mid \lambda)=\sum_{l=0}^{n}\left(\begin{array}{c}
n \\
l
\end{array}\right) \frac{S_{1}(l+r, r)}{\left(\begin{array}{c}
l+r \\
r
\end{array}\right)} \lambda^{n-l} B_{n-l}^{(n-l-r+1)}(x+1) .
$$

\section{On partially degenerate Daehee polynomials}

For $\lambda, t \in \mathbb{C}_{p}$ with $|\lambda t|<p^{-\frac{1}{p-1}}$, we consider the partially degenerate Daehee polynomials denoted by $\tilde{d}_{n}(x \mid \lambda)$ and given by the generating function to be

$$
\frac{(1+t)^{\lambda}-1}{\lambda t}(1+t)^{x}=\sum_{n=0}^{\infty} \tilde{d}_{n}(x \mid \lambda) \frac{t^{n}}{n !} .
$$


When $x=0, \tilde{d}_{n}(\lambda)=\tilde{d}_{n}(0 \mid \lambda)$ are called the partially degenerate Daehee numbers,

$$
\lim _{\lambda \rightarrow 0} \frac{(1+t)^{\lambda}-1}{\lambda t}(1+t)^{x}=\frac{\log (1+t)}{t}(1+t)^{x}=\sum_{n=0}^{\infty} D_{n}(x) \frac{t^{n}}{n !} .
$$

Thus, $\lim _{\lambda \rightarrow 0} \tilde{d}_{n}(x \mid \lambda)=D_{n}(x)$.

In this section, we concentrate on the polynomials obtained by degenerating only the numerator part of Daehee numbers and polynomials. We call these numbers and polynomials partially degenerate Daehee numbers and polynomials. The motivation for this partially degenerate concept comes from the Daehee polynomials with $\lambda$-parameter $D_{n}(x \mid \lambda)$. As we know,

$$
\lim _{\lambda \rightarrow 0} D_{n}(x \mid \lambda)=B_{n}(x)
$$

So, the Daehee polynomials with $\lambda$-parameter $D_{n}(x \mid \lambda)$ are a wonderful degenerating function for Bernoulli polynomials.

From the generating function for the partially degenerate Daehee polynomials (20), we can prove the following theorem similarly to (10) and (11).

Theorem 7 For $n \geq 0$, we have

$$
\tilde{d}_{n}(x \mid \lambda)=\sum_{m=0}^{n}\left(\begin{array}{l}
n \\
m
\end{array}\right) \tilde{d}_{m}(\lambda)(x)_{n-m}
$$

and

$$
\tilde{d}_{n}(x+y \mid \lambda)=\sum_{m=0}^{n}\left(\begin{array}{l}
n \\
m
\end{array}\right) \tilde{d}_{m}(x \mid \lambda)(y)_{n-m} .
$$

From (20), we observe the partially degenerate Daehee numbers. Then we have the following identity:

$$
\begin{aligned}
& \frac{(1+\lambda t)^{\frac{1}{\lambda}}-1}{\lambda t} \int_{\mathbb{Z}_{p}}(1+\lambda t)^{\frac{x}{\lambda}} d \mu_{0}(x) \\
& =\left(\sum_{m=0}^{\infty} \tilde{d}_{m}(\lambda) \frac{t^{m}}{m !}\right)\left(\sum_{l=0}^{\infty} D_{l}(\lambda) \frac{t^{l}}{l !}\right) \\
& =\sum_{n=0}^{\infty}\left(\sum_{m=0}^{n}\left(\begin{array}{c}
n \\
m
\end{array}\right) \tilde{d}_{m}(\lambda) D_{n-m}(\lambda)\right) \frac{t^{n}}{n !} .
\end{aligned}
$$

On the other hand,

$$
\begin{gathered}
\frac{(1+\lambda t)^{\frac{1}{\lambda}}-1}{\lambda t} \int_{\mathbb{Z}_{p}}(1+\lambda t)^{\frac{x}{\lambda}} d \mu_{0}(x) \\
=\frac{(1+\lambda t)^{\frac{1}{\lambda}}-1}{\lambda t} \frac{\log (1+\lambda t)^{\frac{1}{\lambda}}}{(1+\lambda t)^{\frac{1}{\lambda}}-1} \\
=\frac{\log (1+\lambda t)^{\frac{1}{\lambda}}}{\lambda t}=\sum_{l=0}^{\infty} \frac{(-1)^{l}}{l+1} \lambda^{l-1} \frac{t^{l}}{l !} .
\end{gathered}
$$


Therefore, by (21) and (22), we obtain the following theorem.

Theorem 8 For $n \geq 0$, we have

$$
\sum_{m=0}^{n}\left(\begin{array}{l}
n \\
m
\end{array}\right) \tilde{d}_{m}(x \mid \lambda) D_{n-m}(\lambda)=\frac{(-1)^{n}}{n+1} \lambda^{n-1}
$$

Let us take $\lambda=1$ and replace $t$ by $e^{\left(e^{t}-1\right)}-1$ in (20). Then similarly to (15) we have the following theorem.

Theorem 9 For $n \geq 0$, we have

$$
\operatorname{Bel}_{k}(x)=\sum_{m=0}^{k} \sum_{n=0}^{m} \tilde{d}_{n}(x \mid 1) S_{2}(m, n) S_{2}(k, m)
$$

\section{On totally degenerate Daehee polynomials}

For $\lambda, t \in \mathbb{C}_{p}$ with $|\lambda t|<p^{-\frac{1}{p-1}}$, we consider the totally degenerate Daehee polynomials denoted by $d_{n}^{*}(x \mid \lambda)$ and given by the generating function to be

$$
\frac{(1+t)^{\lambda}-1}{\log (1+\lambda t)}(1+t)^{x}=\sum_{n=0}^{\infty} d_{n}^{*}(x \mid \lambda) \frac{t^{n}}{n !} .
$$

When $x=0, d_{n}^{*}(\lambda)=d_{n}^{*}(0 \mid \lambda)$ are called the totally degenerate Daehee numbers. Similar to the previous case, we can check that $\lim _{\lambda \rightarrow 0} d_{n}^{*}(x \mid \lambda)=D_{n}(x)$.

The totally degenerate Daehee numbers and polynomials are constructed by degenerating both numerator and denominator of Daehee numbers and polynomials. From the generating function for the totally degenerate Daehee polynomials (23), we can see that the totally degenerate Daehee polynomials are a Sheffer sequence. And we have the following theorem.

Theorem 10 For $n \geq 0$, we have

$$
d_{n}^{*}(x \mid \lambda)=\sum_{m=0}^{n}\left(\begin{array}{l}
n \\
m
\end{array}\right) d_{m}^{*}(\lambda)(x)_{n-m}
$$

and

$$
d_{n}^{*}(x+y \mid \lambda)=\sum_{m=0}^{n}\left(\begin{array}{l}
n \\
m
\end{array}\right) d_{m}^{*}(x \mid \lambda)(y)_{n-m} .
$$

From (23), we consider the following:

$$
\begin{aligned}
\frac{(1+t)^{\lambda}-1}{\log (1+\lambda t)} & =\frac{(1+t)^{\lambda}-1}{\lambda t} \frac{\lambda t}{\log (1+\lambda t)} \\
& =\left(\sum_{l=0}^{\infty} \tilde{d}_{l}(\lambda) \frac{t^{l}}{l !}\right)\left(\sum_{m=0}^{\infty} B_{m}^{(m)}(1) \frac{\lambda^{m} t^{m}}{m !}\right) \\
& =\sum_{n=0}^{\infty}\left(\sum_{l=0}^{n}\left(\begin{array}{l}
n \\
l
\end{array}\right) \tilde{d}_{l}(\lambda) B_{n-l}^{(n-l)}(1) \lambda^{n-l}\right) \frac{t^{n}}{n !} .
\end{aligned}
$$


By comparing the coefficients of both sides of (24), we have the following result.

Theorem 11 For $n \geq 0$, we have

$$
d_{n}^{*}(x)=\sum_{l=0}^{n}\left(\begin{array}{l}
n \\
l
\end{array}\right) \tilde{d}_{l}(\lambda) B_{n-l}^{(n-l)}(1) \lambda^{n-l}
$$

From (23), we observe the following identity:

$$
\begin{gathered}
\frac{(1+t)^{\lambda}-1}{\log (1+\lambda t)}(1+t)^{x} \int_{\mathbb{Z}_{p}}(1+\lambda t)^{\frac{x}{\lambda}} d \mu_{0}(x) \\
=\left(\sum_{m=0}^{\infty} d_{m}^{*}(x \mid \lambda) \frac{t^{m}}{m !}\right)\left(\sum_{l=0}^{\infty} D_{l}(\lambda) \frac{t^{l}}{l !}\right) \\
=\sum_{n=0}^{\infty}\left(\sum_{m=0}^{n}\left(\begin{array}{c}
n \\
m
\end{array}\right) d_{m}^{*}(x \mid \lambda) D_{n-m}(\lambda)\right) \frac{t^{n}}{n !} .
\end{gathered}
$$

On the other hand, by (5) and (20), we have the following:

$$
\begin{gathered}
\frac{(1+t)^{\lambda}-1}{\log (1+\lambda t)}(1+t)^{x} \int_{\mathbb{Z}_{p}}(1+\lambda t)^{\frac{x}{\lambda}} d \mu_{0}(x) \\
=\frac{t}{(1+\lambda t)^{\frac{1}{\lambda}}-1} \frac{(1+t)^{\lambda}-1}{\lambda t}(1+t)^{x} \\
=\left(\sum_{m=0}^{\infty} \beta_{m}(\lambda) \frac{t^{m}}{m !}\right)\left(\sum_{l=0}^{\infty} \tilde{d}_{l}(x \mid \lambda) \frac{t^{l}}{l !}\right) \\
=\sum_{n=0}^{\infty}\left(\sum_{m=0}^{n}\left(\begin{array}{c}
n \\
m
\end{array}\right) \beta_{m}(\lambda) \tilde{d}_{n-m}(x \mid \lambda)\right) \frac{t^{n}}{n !} .
\end{gathered}
$$

Therefore, by (25) and (26), we obtain the following theorem.

Theorem 12 For $n \geq 0$, we have

$$
\begin{aligned}
& \sum_{m=0}^{n}\left(\begin{array}{l}
n \\
m
\end{array}\right) d_{m}^{*}(x \mid \lambda) D_{n-m}(\lambda) \\
& \quad=\sum_{m=0}^{n}\left(\begin{array}{l}
n \\
m
\end{array}\right) \beta_{m}(\lambda) \tilde{d}_{n-m}(x \mid \lambda) .
\end{aligned}
$$

For $r \in \mathbb{N}$, we define the higher-order totally degenerate Daehee polynomials which are given by the generating function to be

$$
\left(\frac{(1+t)^{\lambda}-1}{\log (1+\lambda t)}\right)^{r}(1+t)^{x}=\sum_{n=0}^{\infty} d_{n}^{*(r)}(x \mid \lambda) \frac{t^{n}}{n !}
$$

When $x=0, d_{n}^{*(r)}(\lambda)=d_{n}^{*(r)}(0 \mid \lambda)$ are called the totally degenerate Daehee numbers of or$\operatorname{der} r$. 
From the generating function, we note that

$$
\begin{aligned}
\sum_{n=0}^{\infty} d_{n}^{*(r)}(x \mid \lambda) \frac{t^{n}}{n !} & =\left(\frac{\lambda t}{\log (1+\lambda t)}\right)^{r}\left(\frac{(1+t)^{\lambda}-1}{\lambda t}\right)^{r}(1+t)^{x} \\
& =\left(\sum_{m=0}^{\infty} B_{m}^{(m-r+1)}(1) \frac{\lambda^{m} t^{m}}{m !}\right)\left(\sum_{l=0}^{\infty} \tilde{d}_{l}^{(r)}(x) \frac{t^{l}}{l !}\right) \\
& =\sum_{n=0}^{\infty}\left(\sum_{m=0}^{n}\left(\begin{array}{c}
n \\
m
\end{array}\right) B_{m}^{(m-r+1)}(1) \lambda^{m} \tilde{d}_{n-m}^{(r)}(x)\right) \frac{t^{n}}{n !} .
\end{aligned}
$$

Thus, we have the following theorem.

Theorem 13 For $n \geq 0$, we have

$$
d_{n}^{*(r)}(x \mid \lambda)=\sum_{m=0}^{n}\left(\begin{array}{l}
n \\
m
\end{array}\right) B_{m}^{(m-r+1)}(1) \lambda^{m} \tilde{d}_{n-m}^{(r)}(x) .
$$

Now, we give a relation for the given numbers and polynomials, which are Daehee, degenerate Daehee, partially degenerate Daehee and totally degenerate Daehee numbers and polynomials as follows:

$$
\sum_{k=0}^{n}\left(\begin{array}{l}
n \\
k
\end{array}\right) D_{k} d_{n-k}^{*}=\sum_{k=0}^{n}\left(\begin{array}{l}
n \\
k
\end{array}\right) d_{k} \tilde{d}_{n-k}
$$

and

$$
\sum_{k=0}^{n}\left(\begin{array}{l}
n \\
k
\end{array}\right) D_{k}(x) d_{n-k}^{*}=\sum_{k=0}^{n}\left(\begin{array}{l}
n \\
k
\end{array}\right) d_{k}(x) \tilde{d}_{n-k}
$$

\section{5 q-Analogues of degenerate, partially degenerate and totally degenerate Daehee polynomials}

Some $q$-analogues of the degenerate Bernoulli polynomials are studied by Kim et al. in [19]. This research influenced us to study $q$-analogues of the degenerate Daehee numbers and polynomials. In this section, we study $q$-analogues of degenerate, partially degenerate and totally degenerate Daehee polynomials. And we give some formulas related to those polynomials.

For $\lambda, t \in \mathbb{C}_{p}$ with $|\lambda t|<p^{-\frac{1}{p-1}}$, the $q$-analogues of degenerate Daehee polynomials denoted by $d_{n, q}(x \mid \lambda)$ are given by the generating function to be

$$
\frac{\log (1+t)}{(q-1)+\frac{q-1}{\log q} \log (1+\lambda t)^{\frac{1}{\lambda}}}(1+t)^{x}=\sum_{n=0}^{\infty} d_{n, q}(x \mid \lambda) \frac{t^{n}}{n !}
$$

When $x=0, d_{n, q}(\lambda)=d_{n, q}(0 \mid \lambda)$ are called the $q$-analogues of degenerate Daehee numbers.

We note that

$$
\lim _{q \rightarrow 1} d_{n, q}(x \mid \lambda)=d_{n}(x \mid \lambda)
$$


and

$$
\lim _{\lambda \rightarrow 0 q \rightarrow 1} d_{n, q}(x \mid \lambda)=D_{n}(x) .
$$

From (9), we have the following theorem.

Theorem 14 For $n \geq 0$, we have

$$
d_{n, q}(x \mid \lambda)=\sum_{m=0}^{n}\left(\begin{array}{l}
n \\
m
\end{array}\right) d_{m, q}(\lambda)(x)_{n-m}
$$

and

$$
d_{n, q}(x+y \mid \lambda)=\sum_{m=0}^{n}\left(\begin{array}{l}
n \\
m
\end{array}\right) d_{m, q}(x \mid \lambda)(y)_{n-m} .
$$

Also, by (8) and (29), we consider the following:

$$
\begin{aligned}
& \frac{\log (1+t)}{(q-1)+\frac{q-1}{\log q} \log (1+\lambda t)^{\frac{1}{\lambda}}}(1+t)^{x} \\
& =\frac{t}{(q-1)+\frac{q-1}{\log q} \log (1+\lambda t)^{\frac{1}{\lambda}}} \frac{\log (1+t)}{t}(1+t)^{x} \\
& =\left(\sum_{m=0}^{\infty} b_{m, q}(x \mid \lambda) \frac{t^{m}}{m !}\right)\left(\sum_{l=0}^{\infty} D_{l} \frac{t^{l}}{l !}\right) \\
& =\sum_{n=0}^{\infty}\left(\sum_{m=0}^{n}\left(\begin{array}{c}
n \\
m
\end{array}\right) b_{m, q}(x \mid \lambda) D_{n-m}\right) \frac{t^{n}}{n !} .
\end{aligned}
$$

Thus, we have the following theorem.

Theorem 15 For $n \geq 0$, we have

$$
d_{n, q}(x \mid \lambda)=\sum_{m=0}^{n}\left(\begin{array}{l}
n \\
m
\end{array}\right) b_{m, q}(x \mid \lambda) D_{n-m} .
$$

From (8) and (29), we note that

$$
\begin{gathered}
\frac{\log (1+t)}{(q-1)+\frac{q-1}{\log q} \log (1+\lambda t)^{\frac{1}{\lambda}}} \int_{\mathbb{Z}_{p}}(1+\lambda t)^{\frac{x+y}{\lambda}} d \mu_{q}(y) \\
=\left(\sum_{m=0}^{\infty} \beta_{m, q}(x \mid \lambda) \frac{t^{m}}{m !}\right)\left(\sum_{l=0}^{\infty} D_{l} \frac{t^{l}}{l !}\right) \\
=\sum_{n=0}^{\infty}\left(\sum_{m=0}^{n}\left(\begin{array}{l}
n \\
m
\end{array}\right) \beta_{m, q}(x \mid \lambda) D_{n-m}(\lambda)\right) \frac{t^{n}}{n !} .
\end{gathered}
$$


On the other hand,

$$
\begin{gathered}
\frac{\log (1+t)}{(q-1)+\frac{q-1}{\log q} \log (1+\lambda t)^{\frac{1}{\lambda}}} \int_{\mathbb{Z}_{p}}(1+\lambda t)^{\frac{x+y}{\lambda}} d \mu_{q}(y) \\
=\left(\sum_{m=0}^{\infty} d_{m, q}(\lambda) \frac{t^{m}}{m !}\right)\left(\sum_{l=0}^{\infty} D_{l, q}(x \mid \lambda) \frac{t^{l}}{l !}\right) \\
=\sum_{n=0}^{\infty}\left(\sum_{m=0}^{n}\left(\begin{array}{c}
n \\
m
\end{array}\right) d_{m, q}(\lambda) D_{n-m, q}(x \mid \lambda)\right) \frac{t^{n}}{n !} .
\end{gathered}
$$

Therefore, by (31) and (32), we obtain the following result.

Theorem 16 For $n \geq 0$, we have

$$
\sum_{m=0}^{n}\left(\begin{array}{l}
n \\
m
\end{array}\right) \beta_{m, q}(x \mid \lambda) D_{n-m}=\sum_{m=0}^{n}\left(\begin{array}{l}
n \\
m
\end{array}\right) d_{m, q}(\lambda) D_{n-m, q}(x \mid \lambda) .
$$

We consider the $q$-analogues of partially degenerate Daehee polynomials by the generating function to be

$$
\frac{(q-1)+\frac{q-1}{\log q} \frac{(1+t)^{\lambda}-1}{\lambda}}{t}(1+t)^{x}=\sum_{n=0}^{\infty} \tilde{d}_{n, q}(x \mid \lambda) \frac{t^{n}}{n !} .
$$

When $x=0, \tilde{d}_{n, q}(\lambda)=\tilde{d}_{n, q}(0 \mid \lambda)$ are called the $q$-analogues of partially degenerate Daehee numbers.

Also, we can see the following:

$$
\lim _{q \rightarrow 1} \tilde{d}_{n, q}(x \mid \lambda)=\tilde{d}_{n}(x \mid \lambda)
$$

and

$$
\lim _{\lambda \rightarrow 0 q \rightarrow 1} \tilde{d}_{n, q}(x \mid \lambda)=D_{n}(x)
$$

From the definition of $q$-analogues partially degenerate Daehee numbers and polynomials in (33), we have the following identities.

Theorem 17 For $n \geq 0$, we have

$$
\tilde{d}_{n, q}(x \mid \lambda)=\sum_{m=0}^{n}\left(\begin{array}{l}
n \\
m
\end{array}\right) \tilde{d}_{m, q}(\lambda)(x)_{n-m}
$$

and

$$
\tilde{d}_{n, q}(x+y \mid \lambda)=\sum_{m=0}^{n}\left(\begin{array}{c}
n \\
m
\end{array}\right) \tilde{d}_{m, q}(x \mid \lambda)(y)_{n-m} .
$$


We can also consider the $q$-analogues of totally degenerate Daehee polynomials by the generating function to be

$$
\frac{(q-1)+\frac{q-1}{\log q} \frac{(1+t)^{\lambda}-1}{\lambda}}{(q-1)+\frac{q-1}{\log q} \log (1+\lambda t)^{\frac{1}{\lambda}}}(1+t)^{x}=\sum_{n=0}^{\infty} d_{n, q}^{*}(x \mid \lambda) \frac{t^{n}}{n !} .
$$

When $x=0, d_{n, q}^{*}(\lambda)=d_{n, q}^{*}(0 \mid \lambda)$ are called the $q$-analogues of totally degenerate Daehee numbers.

We can also see the following:

$$
\lim _{q \rightarrow 1} d_{n, q}^{*}(x \mid \lambda)=d_{n}^{*}(x \mid \lambda)
$$

and

$$
\lim _{\lambda \rightarrow 0 q \rightarrow 1} d_{n, q}^{*}(x \mid \lambda)=D_{n}(x)
$$

From (34), we have the following identities.

Theorem 18 For $n \geq 0$, we have

$$
d_{n, q}^{*}(x \mid \lambda)=\sum_{m=0}^{n}\left(\begin{array}{l}
n \\
m
\end{array}\right) d_{m, q}^{*}(\lambda)(x)_{n-m}
$$

and

$$
d_{n, q}^{*}(x+y \mid \lambda)=\sum_{m=0}^{n}\left(\begin{array}{c}
n \\
m
\end{array}\right) d_{m, q}^{*}(x \mid \lambda)(y)_{n-m}
$$

We give the relation among $q$-analogues of degenerate, partially degenerate, totally degenerate Daehee numbers and polynomials and Daehee numbers and polynomials.

Consider the following:

$$
\begin{aligned}
& \frac{(q-1)+\frac{q-1}{\log q} \frac{(1+t)^{\lambda}-1}{\lambda}}{(q-1)+\frac{q-1}{\log q} \log (1+\lambda t)^{\frac{1}{\lambda}}} \frac{\log (1+t)}{t}(1+t)^{x} \\
& =\frac{\log (1+t)}{(q-1)+\frac{q-1}{\log q} \log (1+\lambda t)^{\frac{1}{\lambda}}} \frac{(q-1)+\frac{q-1}{\log q} \frac{(1+t)^{\lambda}-1}{\lambda}}{t}(1+t)^{x} \\
& =\left(\sum_{m=0}^{\infty} d_{m, q}(x \mid \lambda) \frac{t^{m}}{m !}\right)\left(\sum_{l=0}^{\infty} \tilde{d}_{l, q}(\lambda) \frac{t^{l}}{l !}\right) \\
& =\sum_{n=0}^{\infty}\left(\sum_{m=0}^{n}\left(\begin{array}{c}
n \\
m
\end{array}\right) d_{m, q}(x \mid \lambda) \tilde{d}_{n-m, q}(\lambda)\right) \frac{t^{n}}{n !} .
\end{aligned}
$$

Thus we have the following theorem. 
Theorem 19 For $n \geq 0$, we have

$$
\begin{aligned}
\sum_{m=0}^{n}\left(\begin{array}{c}
n \\
m
\end{array}\right) d_{m, q}^{*}(x \mid \lambda) D_{n-m} & =\sum_{m=0}^{n}\left(\begin{array}{c}
n \\
m
\end{array}\right) d_{m, q}(x \mid \lambda) \tilde{d}_{n-m, q}(\lambda) \\
& =\sum_{m=0}^{n}\left(\begin{array}{c}
n \\
m
\end{array}\right) d_{m, q}^{*}(\lambda) D_{n-m}(x) \\
& =\sum_{m=0}^{n}\left(\begin{array}{l}
n \\
m
\end{array}\right) d_{m, q}(\lambda) \tilde{d}_{n-m}(x \mid \lambda) .
\end{aligned}
$$

\section{Competing interests}

The author declares that they have no competing interests.

\section{Author's contributions}

The author carried out all work of this article and the main theorem. The author read and approved the final manuscript.

\section{Acknowledgements}

The author wishes to express sincere gratitude to the referees for their valuable suggestions and comments.

Received: 23 May 2015 Accepted: 27 August 2015 Published online: 16 September 2015

\section{References}

1. Do, Y, Lim, D: On (h, q)-Daehee numbers and polynomials. Adv. Differ. Equ. 2015, 107 (2015)

2. Kim, DS, Kim, T: Daehee numbers and polynomials. Appl. Math. Sci. 7(120), 5969-5976 (2013)

3. Kim, DS, Kim, T: Identities arising from higher-order Daehee polynomial bases. Open Math. 13(1), 196-208 (2015)

4. Kim, DS, Kim, T, Komatsu, T, Seo, J-J: Barnes-type Daehee polynomials. Adv. Differ. Equ. 2014, 141 (2014)

5. Kim, DS, Kim, T, Lee, S-H, Seo, J-J: Higher-order Daehee numbers and polynomials. Int. J. Math. Anal. 8(6), 273-283 (2014)

6. Park, J-W: On the twisted Daehee polynomials with q-parameter. Adv. Differ. Equ. 2014, 304 (2014)

7. Park, J-W, Rim, S-H, Kwon, J: The twisted Daehee numbers and polynomials. Adv. Differ. Equ. 2014, 1 (2014)

8. Roman, S: The Umbral Calculus. Dover, New York (2005)

9. Kim, DS, Kim, T: A note on degenerate Bernoulli numbers and polynomials associated with $p$-adic invariant integrals on $\mathbb{Z}_{p}$. Appl. Math. Comput. 2015(15), 198-204 (2015)

10. Kim, DS, Kim, T, Kwon, HI, Seo, J-J: Daehee polynomials with q-parameter. Adv. Stud. Theor. Phys. 8(13), 561-569 (2014)

11. Kim, DS, Kim, T, Lee, S-H, Seo, J-J: A note on the lambda-Daehee polynomials. Int. J. Math. Anal. 7(62), 3069-3080 (2013)

12. Kim, T: Barnes' type multiple degenerate Bernoulli polynomials and Euler polynomials. Appl. Math. Comput. 258, 556-564 (2015)

13. Bayad, A, Kim, T: Identities involving values of Bernstein, $q$-Bernoulli, and $q$-Euler polynomials. Russ. J. Math. Phys. 18(2), 133-143 (2011)

14. Gaboury, S, Tremblay, R, Fugère, B-J: Some explicit formulas for certain new classes of Bernoulli, Euler and Genocchi polynomials. Proc. Jangjeon Math. Soc. 17(1), 115-123 (2014)

15. Kim, T, Seo, J-J: A note on the partially degenerate Bernoulli polynomials. J. Math. Anal. 6(4) (2015)

16. Prabhakar, TR, Gupta, S: Bernoulli polynomials of the second kind and general order. Indian J. Pure Appl. Math. 11(10), 1361-1368 (1980)

17. Carlitz, L: Degenerate Stirling, Bernoulli and Eulerian numbers. Util. Math. 15, 51-88 (1979)

18. Kim, T: q-Volkenborn integration. Russ. J. Math. Phys. 9(3), 288-299 (2002)

19. Kim, DS, Kim, T, Dolgy, DV: On q-analogues of degenerate Bernoulli polynomials. Adv. Differ. Equ. 2015, 194 (2015)

20. Kim, T, Lee, S-H, Mansour, T, Seo, J-J: A note on q-Daehee polynomials and numbers. Adv. Stud. Contemp. Math. 24(2), $155-160(2014)$

21. Kim, T: $q$-Bernoulli numbers and polynomials associated with Gaussian binomial coefficients. Russ. J. Math. Phys. 15(1), 51-57 (2008)

22. Kim, T, Kwon, HI, Lee, S-H, Seo, J-J: A note on poly-Bernoulli numbers and polynomials of the second kind. Adv. Differ. Equ. 2014, 219 (2014)

23. Kim, DS, Kim, T: Some identities of Bell polynomials. Sci. China Math. 2015(58) (2015) 